\title{
Transformación e innovación organizacional en la pequeña y mediana empresa - el estrés, cambio, una propuesta para desarrollar capacidades dinámicas
}

Transformation and organizational innovation in small and medium enterprises - stress, change, a proposal to develop dynamic capabilities

Transformação organizacional e inovação nas pequenas e médias empresas stress, no entanto, uma proposta para desenvolver capacidades dinâmicas

Jorge R. Salazar Cantón*

Universidad Autónoma de Yucatán, México jsalazar@uady.mx

Gustavo Barredo Baqueiro**
Universidad Autónoma de Yucatán, México
gbarredo@hotmail.com
Teresa Sabido Dominguez ${ }^{* * *}$
Universidad Autónoma de Yucatán, México
tere.sabido@coreo.uady.mx

\footnotetext{
* Universidad Autónoma de Yucatán, carretera Antigua a Chuburná, domicilio conocido, teléfono 529999100266, jsalazar@uady.mx

** Universidad Autónoma de Yucatán, carretera Antigua a Chuburná, domicilio conocido, teléfono 529999810926 gbarredo@hotmail.com

*** Universidad Autónoma de Yucatán, carretera Antigua a Chuburná, domicilio conocido, teléfono 529999810926 tere.sabido@coreo.uady.mx
} 


\section{Resumen}

La globalización, la competitividad, han traído avances en la ciencia y en la tecnología, que impactan a las personas y por tanto a las organizaciones, lo cual dificulta la capacidad de las personas para adaptarse de una manera adecuada y efectiva a los cambios vertiginosos que provocan dichos eventos.

Lo anterior provoca por tanto tensiones en las personas y por tanto afecta el comportamiento humano, lo cual orienta el interés de identificar, recopilar, reflexionar y por tanto proponer herramientas y/o instrumentos y/o metodologías que ayudar a mejorar y/o adquirir mejores competencias en las personas y por ende a las pequeñas y medianas empresas donde laboran. Asimismo, contar con mejores prácticas que permitan adaptarse a los cambios que el entorno exige.

El presente trabajo versará sobre el proyecto de intervención realizado en una organización comercial mexicanos, con la participación de cuatro equipos conformados por ejecutivos y personal en los procesos clave, con la finalidad de impulsar y promover una manera distinta de resolver problemas, mediante un proyecto de investigación-acción, con estudio de casos. El proyecto está dividido en varias etapas anuales (dos años), y se ha empleado adicionalmente como estrategia para abordar este tema (cambio, estrés y desarrollo de capacidades organizacionales). Se propone una metodología europea adaptada al entorno mexicano, denominada "3-P" propuesta por sus autores Klaus North, Peter Freidrich y Maja Bernhardt, la cual fue desarrollada con éxito en Europa, con el propósito de desarrollar capacidades dinámicas en las organizaciones.

Palabras Clave: Cambio. Estrés, Capacidades dinâmicas.

\section{Abstract}

Globalization and competitiveness, have brought advances in science and technology, which impact people and, therefore, organizations this difficult people's ability to adapt in an appropriate and effective way to the vertiginous changes caused by such events. 
The previous situation causes tensions in people and affects human behavior, which leads the interest of researchers on identifying, collecting, reflecting and therefore proposing tools, instruments and methodologies that help people to improve and acquire better competencies and the transfer them to small and medium enterprises where they work. Is also useful having better practices to adapt the organization to the to the changes environment demands.

This work is focused on an intervention project on a Mexican commercial organization, four teams participated, formed by key processes executives, in order to promote and improve different ways of solving problems, through a project of action-research, with case studies. The project is divided into several annual stages (two years), and has been used as a strategy to address change, stress and organizational capabilities development. A European proven methodology, called “3-P”, developed by Klaus North, Peter Freidrich and Maja Bernhardt is proposed for this study, which has been adapted to the Mexican environment, with the aim of developing dynamic capacities in organizations.

Key words: Change, Stress, Dynamic capabilities

\section{Resumo}

A globalização, a competitividade, trouxeram avanços na ciência e tecnologia, impactando as pessoas e, portanto, as organizações, o que dificulta a capacidade das pessoas de se adaptar de forma adequada e eficaz a mudanças rápidas causar esses eventos.

Isso faz com que, portanto, a tensão nos indivíduos e, portanto, afeta o comportamento humano, que orienta o interesse de identificar, recolher, refletir e, portanto, propor instrumentos e / ou instrumentos e / ou metodologias que ajudam a melhorar e / ou adquirir melhor habilidades de pessoas e, assim, pequenas e médias empresas onde trabalham. Também têm as melhores práticas para se adaptar às mudanças que as exigências ambientais.

Este artigo incidirá sobre o projecto de intervenção realizada em uma organização comercial do México, com a participação de quatro equipes compostas de executivos e 
funcionários em processos-chave, a fim de incentivar e promover uma maneira diferente de resolver os problemas através de um projecto de pesquisa-ação com estudos de caso. $\mathrm{O}$ projeto está dividido em várias etapas anuais (dois anos), e além disso é utilizado como uma estratégia para abordar esta questão (mudança, stress e desenvolvimento da capacidade organizacional). uma metodologia europeia adaptada ao ambiente mexicana, chamada "3P" proposto pelos autores Klaus Norte, Peter Freidrich e Maja Bernhardt, que foi desenvolvido com sucesso na Europa, a fim de desenvolver as capacidades dinâmicas nas organizações é proposto.

Palavras-chave: Mudança. Estresse, capacidades dinâmicas.

Fecha recepción: Enero $2016 \quad$ Fecha aceptación: Junio 2016

\section{Introduccion}

La evolución y mundo actual ante la globalización y la actual crisis mundial están obligando a las organizaciones, a adaptarse y realizar urgentes cambios y transformaciones en su manera de pensar, organizarse, y actuar para poder competir y en muchos casos sobrevivir al agresivo entorno cambiante de una manera vertiginosa. La escasez de recursos tanto económicos como el tiempo requerido para afrontar dichos retos dificultan el cumplimiento de los propósitos organizaciones, ejerciendo presiones adicionales a las personas que laboran en las mismas lo cual se traduce en elementos que impulsan a las tensiones normales de la vida, a las presiones laborales que aunque en algunos casos son motivacionales (el estrés positivo o eutrés ), generalmente, dichas presiones o tensiones son negativas y que afectan de muy diversas maneras a las personas y por tanto al clima de trabajo y a la ejecución de las tareas planeadas.

Es por ello que es importante investigar, reflexionar, entender, comprender y proponer acciones que permitan establecer mejores prácticas así como proponer herramientas nuevas 
y metodologías que ayudarán a lograr o permitir nuevos aprendizajes que ayudarán a lograr dichas adaptaciones.

El presente trabajo versará sobre el trabajo realizado, mediante una intervención en una organización comercial que ha sido observada en un año, mediante un proyecto de investigación-acción, con estudio de caso, en la que se formaron tres equipos de trabajo con personal clave tanto administrativo como operativo y técnico, así como encargados de área.

Se presentará lo que son las capacidades dinámicas, el estrés y su impacto en las personas y por tanto en las organizaciones y como éste dificulta el desarrollo de dichas capacidades, utilizando una metodología europea aplicada con éxito anteriormente y al cual basada en el aprendizaje del equipo de trabajo interventor se han realizado modificaciones para implementar contribuir a desarrollar esas capacidades y permitir las adaptaciones y manejo del estrés, permitiendo resolver y tomar decisiones de una manera más asertiva, efectiva y expedita estableciendo nuevas formas de pensar y actuar y por tanto realizar actividades y procesos innovadores Se presentarán y divulgarán por tanto los avances obtenidos hasta el momento en el proyecto de investigación e intervención propuesto.

\section{CAPACIDADES DINAMICAS}

Asimismo reviste particular importancia hablar de la necesidad de conocer y proponer el desarrollo de las capacidades dinámicas con el propósito de promover su establecimiento en las organizaciones.

¿Cómo impulsar ventaja competitiva en las organizaciones? La teoría de las capacidades dinámicas en las organizaciones trata de dar respuesta a dicha pregunta.

Un enfoque presentado de una manera innovadora lo propone Teece (1997), señalando que las empresas deben de desarrollar, configurar, reconfigurar, reformar buscando innovar para así poder responder a los cambios que el entorno exige. 
También menciona que la empresa debe aprender a generar capacidades en los procedimientos y procesos de la misma para lograr alcanzar ventajas competitivas, que a su vez reforzarán nuevas acciones que ayudan a resolver problemas, tomar decisiones de una manera más efectiva y por tanto una mayor capacidad de adaptación necesaria.

Collís (1994) propone un enfoque de aprender a aprender y establece que las organizaciones deben de trabajar una metacapacidad, y que podrían ser la habilidad para identificar, analizar, evaluar, propones, incorporar y establecer nuevas competencias y/o competencias (Danneels, 2002).

Según Hamel (1994), existen tres grupos de capacidades funcionales claves para poder desarrollar las capacidades dinámicas y que son las relacionadas con la funcionalidad de la empresa, al mercado y a los procesos.

Los conocimientos estratégicos según Birchall (2004), valores culturales, normas, el compromiso y en general el conjunto de los conocimientos y habilidades de los empleados que laboran en las organizaciones, los sistemas organizacionales, técnicos, físicos y las estructuras y procesos son importantes reforzarlos.

Por otra parte es importante la aportación de Meyer (1993), en el sentido que las principales barreras o inhibidores para establecer o desarrollar las capacidades dinámicas pueden ser: el costo del éxito (entendiendo la zona de confort existente - al tratar de mantener acciones y actividades que se realizan cotidianamente- en la organización que impiden realizar nuevas $\mathrm{y}$ diferentes formas de pensar y actuar), la ansiedad provocada por obtener nuevos resultados, las malas prácticas son también importantes.

Es por ello que con el objetivo de las organizaciones de ser más competitivas, están obligadas a tener que adaptarse, modificando sus formas de pensar y actuar, partiendo de la información para analizarla y aprender a generar nuevos conocimientos y habilidades para impulsar nuevas capacidades organizacionales. 


\section{EL ESTRES}

La palabra estrés es una adaptación gráfica del inglés stress, y se utilizó originariamente en la Física para referirse a la fuerza que, aplicada sobre un objeto, hace que este se deforma o se rompa.

La Real Academia Española la define como la "tensión provocada por situaciones agobiantes que originan reacciones psicosomáticas o trastornos psicológicos a veces graves" (Real Academica Española, 2013) y también es utilizado como sinónimo de fatiga, ansiedad, angustia y agotamiento.

Hans Selye, en 1936, llevó el término al campo de la Psicología, al describir el proceso psicológico y fisiológico del individuo que se encuentra sometido a tensiones o presiones con estresores o eventos o acciones que sean causa de impactos en el organismo de las personas que son impactadas. (Seyle, 1976)

La Asociación Americana de Psicología identifica tres tipos de estrés: a) agudo, siendo este el más común y que son las presiones anticipadas del futuro o del pasado reciente, b) el crónico que es permanente y dañino y pernicioso para el ser humano porque destruye mente cuerpo y vida. Lo padecen con frecuencia los estratos socioeconómicos más bajos, o familias disfuncionales o aquellas personas que hayan padecido algún evento traumático (ataque al corazón, estados emocionales irritables permanentes o depresiones frecuentes) (American Psychological Association, 2013)

Por su parte la Organización Mundial de la Salud (OMS) lo define como "la reacción que puede tener el individuo ante exigencias y presiones laborales que no se ajustan a sus conocimientos y capacidades y que ponen a prueba su capacidad para afrontar la situación". (Salud, 2004)

Es importante mencionar que existen dos tipos de estrés (el bueno o positivo) o llamado eutrés que consiste cuando el empleado o trabajador tiene un alto grado de motivación, está muy orientado a los resultados y en activación constante y generalmente sucede cuando la 
organización ha proporcionado los elementos, implementos y herramientas necesrios para el cumplimiento de las tareas y metas. Este tipo de estrés impulsa la productividad de la persona que lo manifiesta. Pero cuando esa tensión se eleva y afecta el comportamiento de la persona de tal manera que afecta a su salud surge el segundo tipo de estrés que es el llamado estrés malo o "distres" (Fidalgo, 2011)

Ejemplos de lo anteriormente mencionado podemos citar la vitalidad, entusiasmo, optimismo, resistencia a las enfermedades, vigor físico en el caso de eutrés, así como fatiga, irritación, falta de concentración, baja productividad y creatividad en el caso del distrés o estrés laboral para efectos del presente trabajo.

Los efectos que el estrés provoca en el individuo como se ha visto en los ejemplos antes citados producen en sí consecuencias de tipo psicológico, fisiológico (cardiopatías, colon irritable por ejemplo), traen inevitablemente consecuencias e impactos en las organizaciones como por ejemplo, inasistencia, impuntualidad, falta de productividad, contribuye de una manera importante a la rotación de personal, aumento en las tasas de accidentes laborales etc. Organización Mundial de la Salud. (Salud, 2004)

Estos efectos, generalmente son causados por dos grandes motores: el propio individuo y su interrelación con los demás y la propia organización vista como un sistema de personas organizadas mediante un contrato (Salazar, 2011). Veamos brevemente porqué.

\section{- Causas que originan el estrés}

La Organización Mundial de la Salud, (2004) definió que existen varias causas en este sentido en su informe relacionado con el estrés:

Un mal clima laboral y las malas relaciones entre compañeros de trabajo, el mob o acoso laboral, la ausencia o deficiencia en los procedimientos para resolver problemas, los salarios insuficientes para cubrir las necesidades básicas, la falta de desarrollo profesional o promociones adecuadas, las presiones familiares, la falta de apoyo de los mismos ante las 
situaciones que el centro de trabajo dispone, falta de reconocimiento, inadecuados liderazgos, mala comunicación, entre otras razones provocan tensiones en el trabajador que pueden ocasionar un mal estrés. (Salazar, 2011).

Por tanto el estrés tiene una doble vía, la de los trabajadores en las Organizaciones, dada sus circunstancias personales, familiares y como producto de la interrelación entre compañeros de trabajo, así como la propia entidad que genera impulsores que impulsan el estrés y por tanto ambos merman la productividad y rentabilidad en las Organizaciones.

Por tanto el estrés es una barrera para generar capacidades dinámicas en la Empresa.

\section{LA METODOLOGIA 3P DEL DR KLAUS NORTH Y SUS COLEGAS}

La metodología 3P (Prevención Participativa del Personal hospitalario), propuesta por el Dr. Klaus North y sus colegas (North, 2009), se centra en lograr aprendizajes y cambios organizacionales, mediante una reducción de la carga de trabajo revisando los procesos y procedimientos en la Organización, atención de calidad al cliente y elevando la productividad personal reduciendo por tanto el estrés y organizando equipos de trabajo cooperativos y colaborativos

La metodología propuesta incorporando las aportaciones del equipo mexicano denominando CREAANLO (Creación, Asistencia y Análisis de las Organizaciones) de la Facultad de Contaduría y Administración de la Universidad Autónoma de Yucatán se basa principalmente en llevar un proceso participativo de mejora continua, establecer un procedimiento estructurado en la que se identifiquen las principales causas y su reducción o eliminación del estrés, mediante el establecimiento y aplicación de seis principios, así como darle un seguimiento y documentado de las propuestas de mejora todo ello con el apoyo de la alta dirección. 
Los principios en que se basa esta metodología para reducir el estrés, propuesta son:

1. - En reuniones establecidas de una manera diferente, se acuerdan en equipo prioridades y establecen tareas a realizar

2. - Estar conscientes y tener planes alternativos ante las contingencias para poder manejar aquellas situaciones imprevistas.

3. - Tener siempre una comunicación plena, simple, sencilla, y sobre todo claras abiertas

4. - Generar una forma de trabajar y una nueva competencia mediante el establecimiento de equipos de trabajo colaborativos

5. - Una clara definición de lo que significa una buena atención al cliente y unificar objetivos y procedimientos

6. - Tomar pausas y establecer estrategias de descanso necesarias. (North, 2009).

\section{METODOLOGIA}

El proyecto de intervención tuvo una duración de diez meses. Inició en noviembre de 2014 y concluyó en agosto de 2015. Esta ha sido la primera etapa de dos y se presentan los avances del mismo.

Se realizó un proyecto investigación cualitativa, mediante el estudio de caso y el método de investigación-acción, en los que se observaron a cuatro equipos de personal clave de la organización (técnicos, administrativos y responsables de área), objeto del estudio.

Se llevaron y establecieron protocolos. Se realizaron entrevistas, reuniones de trabajo, se grabaron en audio y video las sesiones para recoger la información. Se estableció, una matriz con la comparación de las observaciones e informaciones recabadas en cada hospital y una general para los tres hospitales observados. Se establecieron categorías así como listas de verificación. Se presentan cuadros que sintetizan las observaciones realizadas.

Se realizaron reuniones inicialmente con una periodicidad semanal, posteriormente quincenal y finalmente mensual estableciendo una agenda de trabajos metas, acciones y responsables definidos. Es una entidad comercial dedicada a la compraventa de insumos de 
aseo, limpieza y servicios de seguridad. Se analizaron, propusieron proyectos a realizar de mejora, orientados a reducir cargas e incrementar los resultados sin considerar estímulos y castigos por los logros o no obtenidos.

\section{PROBLEMAS IDENTIFICADOS Y RESULTADOS}

Como se podrá observar los principales problemas que impulsan el estrés en los equipos de trabajo observados son problemas a la organización del trabajo y sus procesos $(46 \%)$, su deficiente comunicación, la configuración, integración y operación de verdaderos trabajos en equipo, clima organizacional deficiente (54\%), que contribuyen principalmente a la dificultad en la toma de decisiones y obtención de resultados y que fueron considerados factores de estrés. 


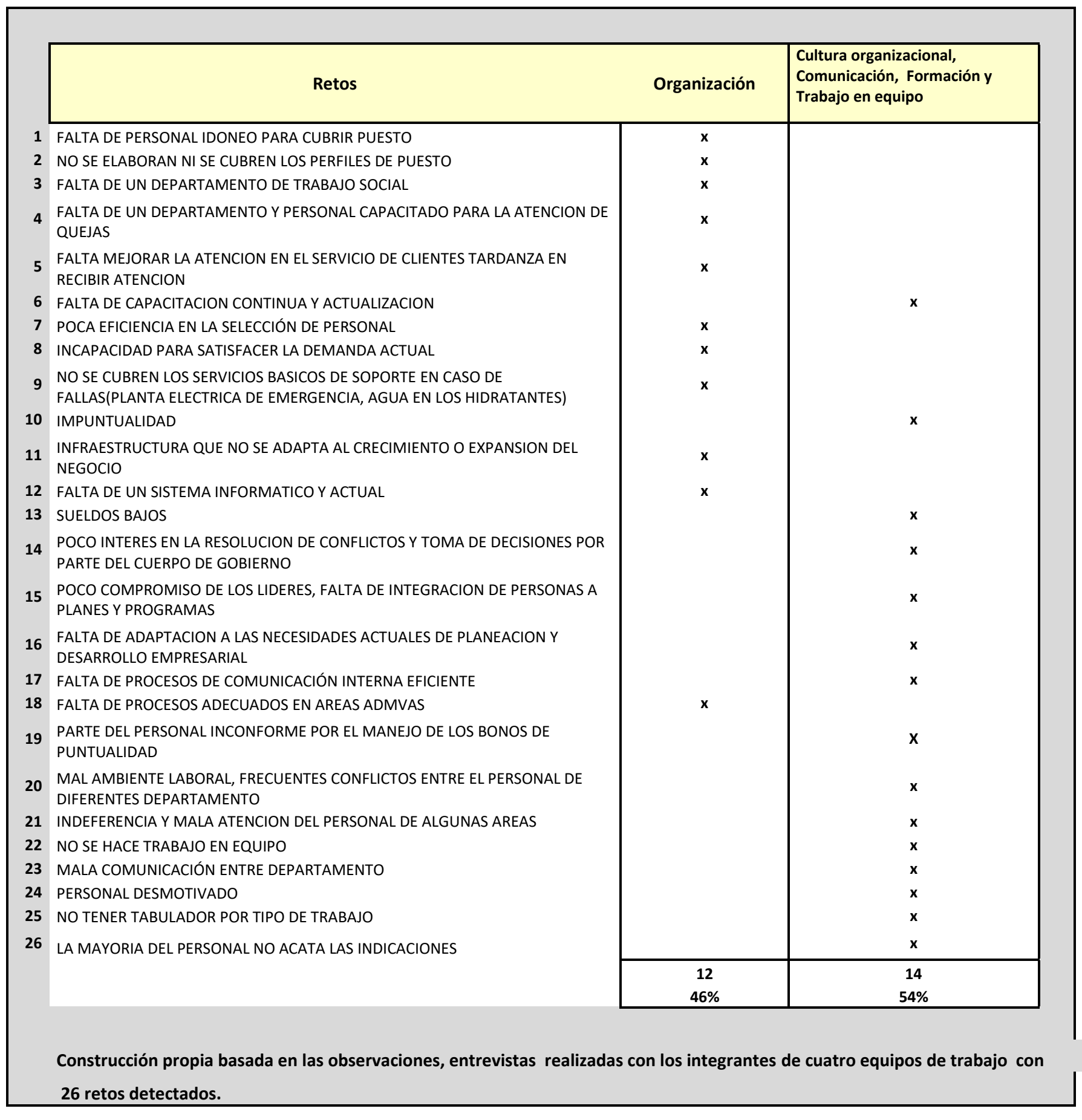




\section{RESULTADOS}

Ante la deficiente integración de trabajos en equipos cooperativos y colaborativos, un clima organizacional tenso y bajo constante presión, la mala comunicación existente interdepartamental, la falta de puntualidad y ausentismo, las indiferencias detectadas generando una falta de interés y motivación, abusos de autoridad, falta de apoyo de los responsables aunado a la falta de una claridad de funciones, tareas, responsabilidades y metas esperadas tanto por parte de las autoridades como de los empleados, traen como como consecuencia cargas tensionales que son barreras constantes al logro de resultados por el estrés provocado en los individuos.

La sumatoria de esfuerzos individuales al logro de los grupos de trabajo contribuye a tener resultados -en su caso- bajo fuertes presiones y tensiones.

\subsection{Establecimiento de nuevos procesos y proyectos}

De una manera cooperativa y colaborativa se estableció un proyecto de reorganización tanto que tuvo como resultados relevantes

- Establecimiento de un departamento de personal y una oficina de atención a los clientes y proveedores.

- Reasignación y reorientación de funciones

- Dada la revisión de los procesos y procedimientos permitió una agilización de procesos que redundaron en simplificadores de las mismas, así como facilitar el logro de resultados y evitando los conflictos interdepartamentales

- Se redujeron las quejas de clientes

- Asimismo los procesos nuevos que se crearon -por ejemplo en materia de suministros de insumos o de seguridad mediante la creatividad y la innovación para la solución de los retos permitieron significativamente reducir los conflictos y mejora en los resultados.

- Se mejoraron los indicadores en tiempos atención y satisfacción del usuario. 


\subsection{Impuntualidad y asistencia}

En los cuatro equipos de trabajo participantes los indicadores de puntualidad iniciales y retraso arrojaban 787 minutos y lo revelador es que en las primeras seis semanas de intervención sin que se establecieran ni estímulos ni castigos como premisas iniciales, sino que como consecuencia con la reflexión y concientización y con acciones no coercitivas ni estimulantes se redujeron a 18 minutos. A las nueve semanas el indicador llegó a cero.

\subsection{Reorganización y mejoras.}

Como consecuencia en la mejora del clima organizacional, al disminuir los conflictos, mejorar la comunicación y los procesos y el establecimiento de verdaderos equipos de trabajo participativos, cooperativos y colaborativos contribuyeron a incrementar y mejorar los resultados propuestos y reduciendo las tensiones entre los participantes.

El establecimiento de nuevas formas de abordar, analizar, resolver y ejecutar las tareas y acciones para resolver los retos, producto del aprendizaje obtenido basado principalmente en una nueva forma de percibir y pensar y actuar generando un conocimiento organizacional nuevo y satisfactorio.

\section{Reflexiones y conclusiones}

¿Las metodologías aplicadas, han contribuido o ayudado a la construcción de una metodología que impulse a la generación de actividades de innovación, a ayudar a "pensar" inteligentemente, a innovar a las organizaciones (desarrollar capacidades dinámicas organizacionales)? ¿Cómo se interrelacionan el estrés, el desarrollo de capacidades dinámicas y la transformación organizacional? Son las preguntas permanentes que el equipo de trabajo mexicano involucrado se plantea alrededor de este tipo de proyectos.

Tratemos de dar una explicación a estos planteamientos y sus posibles respuestas que tanto necesitan y requiere nuestra sociedad tanto empresarial como académica. 
Las percepciones que un individuo o persona tenga de la realidad resultado de sus antecedentes, circunstancias y competencias previas vividas y adquiridas por la persona, y que hacen que las tensiones que el entorno que le rodea cause, impulse e incluso exacerbe dichas motivaciones positivas (eutrés) que al salirse de proporción genere un distress (estrés malo), son un primer elemento a considerar.

Un segundo elemento es la propia Organización que impulsa, causa o sobreestimule la tensión generando estrés como consecuencia de liderazgos, comunicación, procesos, estructuras, falta de verdaderos equipos de trabajo, cultura organizacional deficiente, insuficiente o no adecuados generan cargas estresantes a las personas que colaboran en ella

\section{Impactos.-}

Por tanto el impacto en la salud del individuo, la afectación en los resultados e indicadores que generen valor, la generación de barreras que impulsan la innovación en los procesos y las estrategias que son un fuerte obstáculo en las capacidades dinámicas en las organizaciones. Generando además del estrés, frustraciones en las personas y por ende círculos y/o bucles perversos.

En la Organización es motivo de resistencias, conflictos, dificultades en la formación de verdaderos equipos de trabajo cooperativos, colaborativos de alto desempeño, que son barreras hacia la aportación de nuevas ideas, productividad, nuevas formas de trabajo y resultados que permitan el logro del propósito, visión, objetivos y estrategias en la Organización

Por tanto el estrés tiene altos impactos en los equipos de trabajo, la productividad, innovación y rentabilidad empresarial afectando a las personas en sus vidas.

Y a lo anterior podríamos agregar las exigencias e impactos del entorno que presiona a los directivos en sus formas de liderar sobretodo si no se conocen los nuevos roles y la importancia de la delegación, de dar libertad para actuar, de empoderar a los empleados, y 
realizar por tanto los ajustes necesarios para impulsar la innovación en las organizaciones entonces podríamos tener la explicación del porque el estrés impide el desarrollo de capacidades dinámicas organizaciones que faciliten esa adaptación necesaria ante los cambios.

Al no tener y formas esas nuevas o fortalecer las existentes capacidades organizacionales, que generen un valor agregado, que permitan una organización inteligente que aprenda a aprender y donde el error por ejemplo no sea mal visto sino una oportunidad para aprender, en donde se generen nuevas formas de liderazgo, nuevos procesos que den como resultado procesos innovadores por ejemplo.

Lo anterior traerá como consecuencia que la organización no pueda transformarse.

La metodología utilizada ha ido adaptándose a nuestro entorno, incorporando elementos antes no estudiados como lo son los temas aquí propuestos e incorporando elementos que la metodología inicial no tenía.

Los resultados y sus impactos están a la vista.

En las organizaciones mexicanas intervenidas al lograr concientizar, reflexionar, discutir y asimilar estas cualidades, han permitido transformaciones ya que, por una parte formar verdaderos equipos de trabajo participativos y colaborativos, nuevos enfoques de liderazgo, establecer nuevas visiones, percepciones, procesos en la forma de hacer las cosas que han permitido disminuir el estrés y por tanto incrementar la productividad en indicadores que van del 60 al 80\%, probando y aprobando la metodología descrita y el proceso de intervención que ha permitido a las organizaciones observadas generar capacidades dinámicas que antes no tenían.

El reto ahora es que el esfuerzo por un lado llegue a toda la estructura en cada equipo de trabajo en cada organización intervenida que se mantenga el esfuerzo y no pierda ese impulso inicial que el proyecto y la metodología han establecido sino también que estas 
bondades del proyecto lo reciban otras organizaciones y poder contribuir e impulsar un cambio en el sector observado y/o intervenido.

Por tanto lo que el proyecto está construyendo (no hay que olvidar que es el primer año de implementación) es una alternativa para las organizaciones pequeñas y medianas en nuestro país para cambiar, para generar esas capacidades organizacionales que hoy día se requieren.

\section{Bibliografía}

American Psychological Association. (15 de Julio de 2013). American Psychological Association. Obtenido de http.//www.apa.org/centrodeapoyo/tipos.aspx

Birchal, D. (2004). Market understanding as a determinant of innovation sucess in SMEs. EBS Reviex, 17-29.

Collis, D. (1994). Research Note- How Valuable Are Organizational Capabilities. Strategic Management Journal, 143-152.

Danneels, E. (2002). The dynamics of product innovation and firm competences. Management Journal, 1095-1121.

Fidalgo, V. (2011). El estrés laboral. Barcelona: De Vecchi ediciones S.A.

Hamel, G. \&. (1994). Competing for the Future. Harvard Business Review, 72(4): 122-128.

Klaus, N. \&. (2009). Die Gesundheitshebel. Wiesbaden: Gabler.

Meyer, M. (1993). The Product Family and the Dynamics of Core Capability. Sloan Management Reviex, 34(3):29-47.

Real Academica Española. (15 de Julio de 2013). Real Academica Española. Obtenido de http://www.rae.es/rae.html

Salazar, C. (2011). Le changement dans les hopitaux mexicains;proposition d"un modele integral d“intervention. A partir du cas de l'Hospital de la Amistad dans l'Etat de Yucatan Mexique. Bayonne, Francia: Universidad de Pau. 
Salud, O. M. (2004). La organización del trabajo y estrés. Ginebra, Suiza: OMS.

Seyle, H. (1976). The stress of life. Nueva York: Mc Graw Hill.

Teece, D. \&. (1997). Dynamic Capabilities and Strategic Management. Management Journal, Vol18 No 7, pp509-533. 